\title{
Classification of Diabetic Retinopathy Images by Using Deep Learning Models
}

\author{
Suvajit Dutta, Bonthala CS Manideep, Syed Muzamil Basha, \\ Ronnie D. Caytiles ${ }^{1}$ and N. Ch. S. N. Iyengar ${ }^{2}$ \\ SCOPE, VIT, Vellore, India \\ ${ }^{1}$ Multimedia Eng., Dept, Hannam University, Daejeon, Korea \\ ${ }^{2}$ Dept. of IT, Sreenidhi Institute of Science and Technology, Yamnampet, \\ Ghatkesar, Hyderabad, TS \\ srimannarayanach@sreenidhi.edu.in
}

\begin{abstract}
Diabetes or more precisely Diabetes Mellitus (DM) is a metabolic disorder happens because of high blood sugar level in the body. Over the time, diabetes creates eye deficiency also called as Diabetic Retinopathy (DR) causes major loss of vision. The symptoms can originate in the retinal area are augmented blood vessels, fluid drip, exudates, hemorrhages, and micro aneurysms. In modern medical science, images are the indispensable tool for precise diagnosis of patients. In the meantime evaluation of contemporary medical imageries remains complex. In recent times computer vision with Deep Neural Networks can train a model perfectly and level of accuracy also will be higher than other neural network models. In this study fundus images containing diabetic retinopathy has been taken into consideration. The idea behind this paper is to propose an automated knowledge model to identify the key antecedents of DR. Proposed Model have been trained with three types, back propagation NN, Deep Neural Network (DNN) and Convolutional Neural Network (CNN) after testing models with CPU trained Neural network gives lowest accuracy because of one hidden layers whereas the deep learning models are out performing NN. The Deep Learning models are capable of quantifying the features as blood vessels, fluid drip, exudates, hemorrhages and micro aneurysms into different classes. Model will calculate the weights which gives severity level of the patient's eye. The foremost challenge of this study is the accurate verdict of each feature class thresholds. To identify the target class thresholds weighted Fuzzy C-means algorithm has been used. The model will be helpful to identify the proper class of severity of diabetic retinopathy images.
\end{abstract}

Keywords: Diabetic Retinopathy (DR), Retina, Classification, Threshold, Deep Learning (DL), Deep Neural Network (DNN), Convolutional Neural Network (CNN)

\section{Introduction}

Diabetes is the dissolute epidemic universally, specifically in the Indian society. This is leading to an overabundance of Diabetes which spreads syndromes like DR. It is necessary to develop an automated diagnostic model to expand the work of the Ophthalmic and condense morbidity of the patients. Diabetes affected to 422 million people worldwide; India orders among top 3 countries by means of the diabetic population. In recent years it enlarged from 108 million - 422 million half population are living in India, China, USA, Brazil and Indonesia [42]. Centered on the Lancet study in China, India, and the USA [43] are the top three countries with the high number of diabetic population. Diabetes gives rises to many problems, and

Received (October 8, 2017), Review Result (December 15, 2017), Accepted (December 22, 2017) 
among them the paper focuses on Diabetic Retinopathy, which relates to the loss of vision. DR occurs due to the presence of high glucose level in blood, resulting in damaging the small blood vessels present in the retina. The consequence of which is extra fluid, blood, cholesterol along with the other fats in retina, resulting in thickening and swelling of the macula [14]. In process of supplying sample blood, retina starts growing some new abnormal delicate blood vessels called IrMAs (Intraretinal Microvascular Abnormalities)[44]. The augmented pressure in the eye may result in damaging of the optic nerve in late stages.

DR can hence be stated briefly as the damage caused to retinal blood vessels because of complications of the diabetes, leading to the loss of vision(which in this case is ir-riversible) subsequently. Retinal screening is a possible solution for diagnosis of the damaged caused to retina at initial stages[5]. Diabetic Retinopathy is asymptotic initially and hence most of the patients remain unaware of the condition unless it have emotional restricts their vision. Therefore, prior and consistent screening of Diabetic Retinopathy is essential in order to avoid further difficulties and to control progression of disease. A key symptom of DR is exudates [15], which may be bagged in the fundus image of the eyes and is a sign of developing DR in the patient or he may already has developed it. Discovery of lesions in the fundus images may also contribute in the detection of Diabetic Retinopathy at early stage. [7] To study DR, ophthalmologists often consider the color fundus images, considering various feature elements related to Diabetic Retinopathy, such as hemorrhages, soft and hard exudates and micro aneurysms (MA) which are mini chambers like arrangements induced by local distension of the capillary walls, visible as tiny red dots.

Diabetic retinopathy may progress through four stages: (i) Mild non-proliferative retinopathy, which relates to the occurrence of micro aneurysms (MA) and is regarded as the earliest stage. (ii) Moderate non-proliferative retinopathy, where the blood vessels nourishing the retina might distort and swell with the progress of disease, losing their ability of blood transportation. (iii) Severe non-proliferative retinopathy, results in deprived blood supply to retina due to the blockage of more number of blood vessels, hence signaling the retina for growing of fresh blood vessels. (iv)Proliferative diabetic retinopathy (PDR), is the advanced stage, where the growth features secreted by the retina activate proliferation of the new blood vessels, growing along inside covering of retina in some vitreous gel, filling the eye. Newly created blood vessels are friable, making them bleed and leak more often. Moreover, the associated scar tissue may contract thus causing retinal detachment, leading to perpetual vision loss[16].

To study and overcome these problems, the paper focuses on to get optimal model through machine learning, which will include the classical Neural Networks(NN), Deep Neural Networks(DNNs) and the Convolution Neural Networks(CNNs). The neural networks inherit the concept of a biological brain. The outcomes are often hard to achieve as the biological neurons are more complex than these artificial ones, then also researchers have succeeded to some levels. The neural networks performed well but with increased complexity and data size the need for advanced techniques produced, bringing the concepts of deep learning to existence. Prior to deep learning, the hierarchical attribute learning approaches came to light, but due to the problems such as vanishing gradient, started losing their aura as while tracing back to the features it often becomes difficult to get the desired results. The deep learning models, like DNNs and CNNs delivered solutions to overcome this problem of gradient descent. Fuzzy C-Means clustering also comes to picture here, as if the data have any missing labels when the Image Classification will be done at the later stages. So, in order to predict the labels, Fuzzy C - Means [17] is applied so as to find the clusters. 
For further proceeding, these mechanisms are followed by image preprocessing and classification for enhancing the accuracy of the later steps. The preprocessing of image starts with conversion of given image features to gray scale so as to carry out the further analysis. The imaging classification steps works for classifying the images according to the listed features, thus getting the desired results. For the work considering Diabetic Retinopathy, for [6] the image-based tactics, the provided retina image may be divided in several small sub-images. The work also involves reduction of noise as well for obtaining enhanced image and accurate data. The paper discusses about median filter, which is nonlinear digital filtering procedure, used for removing noise in order to provide improved results. The method is preferred as under definite conditions, the filter conserves edges though removing noise. The key idea behind median filter is to operate via signal one entry followed by another, switching every entry with the median of the neighboring entries. If the window (i.e. pattern of neighbor) has odd amount of the entries, then median is middle value when all entries in window are numerically sorted whereas, for some even number of the entries, more than one medians can exist. Precisely, in spite of replacing a pixel value with mean value (like in Mean Filter method for noise reduction regarding image processing) of its neighboring pixels, it substitutes with median of the values. The work included Edge detection among others as edge detection aims identifying points in an image, where there is sharp change in brightness of the image or has some discontinuities. The existing ways of performing edge detection, can be grouped in two broader categories that are the Gradient method and Laplacian method. The gradient technique detects edges by observing the minimum and the maximum in first derivative of image whereas, Laplacian technique searches for the zero-crossings present in second derivative of image in order to find edges. These concepts wrap up the work progressed while image processing.

\section{Literature Review}

Much work has been done by researchers in the field of Diabetic Retinopathy, depending on their research area and field of interest. The related work in the field of medical sciences as well as machine learning, shows that researchers have proposed and implemented various machine learning methods, but the study comparative study among these deep learning methods is still lacking for as far as Diabetic Retinopathy is concerned. The work done hence proves to be a novel approach while considering the results and fining for various machine learning algorithms for DR.

Raman et al. [1]focuses on developing of computer-aided detection mechanism for finding abnormality of the retinal imaging, while detecting the existence of abnormal features from the retinal fundus images. Their proposed methodology focuses on enhancing images, filtering of the noise, detection of the blood vessels and identifying optic disc, extracting exudates and the micro aneurysms (MA), extracting features and classifying various stages of the diabetic retinopathy as mild, the moderate, the severe NPDR(Non-Proliferative Diabetic Retinopathy) and the PDR(Proliferative Diabetic Retinopathy) by the use of machine learning techniques.[2] Singh\&Tripathi used Image analysis techniques for the automated and early discovery of the Diabetic Retinopathy, by the use of Image processing among many other analysis techniques. Soomro et al. in their research, proposed an image enhancement technique on the basis of [3] morphological operation accompanied bythe proposed threshold centered static wavelet transforms for the retinal fundus image in addition to CLAHE (Contrast Limited Adaptive Histogram Equalisation) for vessel enhancement. [4] Zhao et al. in their paper, proposed a novel saliency-based technique for detecting of the leakage in the fluoresce in angiography. Their proposed methodology is validated using only two publicly 
accessible datasets which are Diabetic Retinopathy and Malarial Retinopathy. Prasad et al. proposed use of the morphological operations along withthe segmentation procedures for detecting the blood vessels, micro aneurysms and the. The [5] PCA (Principal Component Analysis) is applied for improved feature selection. Further, Back-propagation $\mathrm{NN}$ and the one-rule classifier methods were deployed for classification of images as non-diabetic or diabetic.M. Usman Akram et al [6] approach is based on a hybrid classifier detecting the retinal lesions by preprocessing, extracting lesions from candidate, formulation of feature followed by classification. The work leads to further extension of m-Mediods based modeling methodology, combining it with Gaussian Mixture Model so to form some hybrid classifier in order to improve accuracy of classification. Winder, R. John, et al [7] survey based on algorithms for automatic detection of retinopathy while considering digital color retinal images. The algorithms considered for study were categorized in 5 stages (preprocessing, localization and segmentation of the optic disk, segmentation of the retinal vasculature, localization of the macula and fovea, localization and segmentation of retinopathy). Haleem, Muhammad Salman, et al [8] a paper surveys advanced methods for automatic extraction of the anatomical features in order to assist the early diagnosis of Glaucoma, from the retinal images. They carried out critical estimations of existing automatic extraction approaches based on the features comprising of CDR (Optic Cup to Disc Ratio), RNFL (Retinal Nerve Fiber Layer), and PPA (Parapapillary Atrophy) among others. This added value for the efficient feature extraction methods relating to the Glaucoma diagnosis. M.R.K. Mookiah et al. [41] has compared multiple method of DR image detection. From the paper a full overview of multiple technique regarding DR image detection has been found. The paper tabulated possible technique and their accuracy results very precisely.

Gardner et al. worked on determining that if neural networks are able to detect the diabetic features prevailing in the [9]fundus images along with comparing network against ophthalmologist screening set of the fundus images. The work showed the detection of hemorrhages, exudates and vessels. Further comparing with ophthalmologist, their network attained better accuracy for detection of the Diabetic Retinopathy. Roychowdhury et al. in their paper, contributed to the reduction of number of features to be used for the [11] lesion classification by the feature ranking by the use of Adaboost. They proposed novel two-step hierarchical classification approach where non-lesions or the false positives are discarded in first step. And in second step, bright lesions are further classified as the hard exudates along with the cotton wool spots. Also red lesions remain classified as the hemorrhages and the micro-aneurysms (MA).[13] Rakshitha et al. work throws light on uses of the new imaging transformation methods for the enhancement of the retinal images like contourlet transform, curvelet transform and wavelet transform. Their paper thus draws comparison among these three imaging transformations. Vo et al. research studies discriminant texture features which are obtained by the color multi-scale uniform [14] LBPs(Local Binary Patterns)that are descriptors on the two proposed hybrid and the five common color spaces. Then the extracted features can be evaluated by enhanced EFM, a Fisher Linear Discriminant.

\section{Proposed Work}

The problem with the other models is mainly preprocessing of the images and model building patterns. Most of the studied literature lacking the image pre-processing steps which might leads to erroneous result. This study is considered on preprocessing of images through various filter mechanisms which enhances the features of the image. Another approach discussed in this study about extracting statistical features out of the 
image, the idea was to extract information out of the resized image of $2000 \times 2000$, because of high resolution gives better exploration. A problem of image training can arise because of image resize factor, because of lack of computation power in systems the image are resized in some of the papers, because of this feature loss factor can arise and the image labels can varied. To overcome this problem Fuzzy C-means clustering (FCM) has been used, the idea behind using FCM is to find out cluster levels of the training data that leads to better training accuracy. Feed Forward-NN, DNN and CNN all having different capabilities in terms of training and predicting, a comparison of three models will give the proper adaptation of model. Both images and statistical data are trained with all neural network model, to justify the difference of using statistical method and Image processing method. FCM has been used in both cases to identify the class labels.

Table 1. Methodology Flow

\begin{tabular}{ll}
\hline & Model Flow Steps \\
\hline & $\begin{array}{l}\text { Extracted the statistical features from unprocessed RGB images. } \\
\text { (average, median, standard deviation, skewness, root mean square } \\
\text { error, mean absolute deviation, quartiles, minimum, maximum \& } \\
\text { threshold level) }\end{array}$ \\
& RGB image taken and converted into grayscale for image filtering \\
& (Median filter, Morphological processing), applied edge detection \\
& for feature extraction from images and binary conversion of image \\
& to highlight all feature. \\
Step 2: & $\begin{array}{l}\text { First Statistical data taken into consideration for image } \\
\text { classification with Feed Froward Neural Network (FNN) for } \\
\text { classification, After that Deep Neural Network (DNN) performed } \\
\text { and compared the result with FNN. }\end{array}$ \\
Step 3: & $\begin{array}{l}\text { Secondly image classification done on processed images with Feed } \\
\text { forward Neural Network (FNN) model and Deep Neural Network } \\
\text { (DNN) model. }\end{array}$ \\
Step 4: & $\begin{array}{l}\text { Performed Convolutional Neural Network (CNN) on processed } \\
\text { images (VGG16 model) }\end{array}$ \\
Step 5: & $\begin{array}{l}\text { Both the result of Step 3, Step 4 and Step 5 have been compared as } \\
\text { per performance and accuracy measurements with testing image set. }\end{array}$ \\
\hline
\end{tabular}

\section{Methodology}

The data set we have used are FUNDUS images [22] of human retina having pixels over $2000 \times 3000$. The dataset downloaded from website kaggle.com [33-38], its being freely available in the website, dataset having over 35000 image for training and 15000 image for testing. The problem with the dataset is its resolution, its fluctuating for different images and the image itself contains noise so proper filtering is necessary to extract proper dataset. For this study 2000 images has been chosen at 7:3 training and testing ratio. Figure 2a. shows the normal retina image without any diabetic sign Figure $2 \mathrm{~b}$. shows the severe diabetic retinopathy image with the all features like Hemorrhages, Hard Exudates, Cotton Wool Spots, and Abnormal Blood Vessels. Figure 2c.and 2d. shows the histogram distribution of the two types of retinal images. 


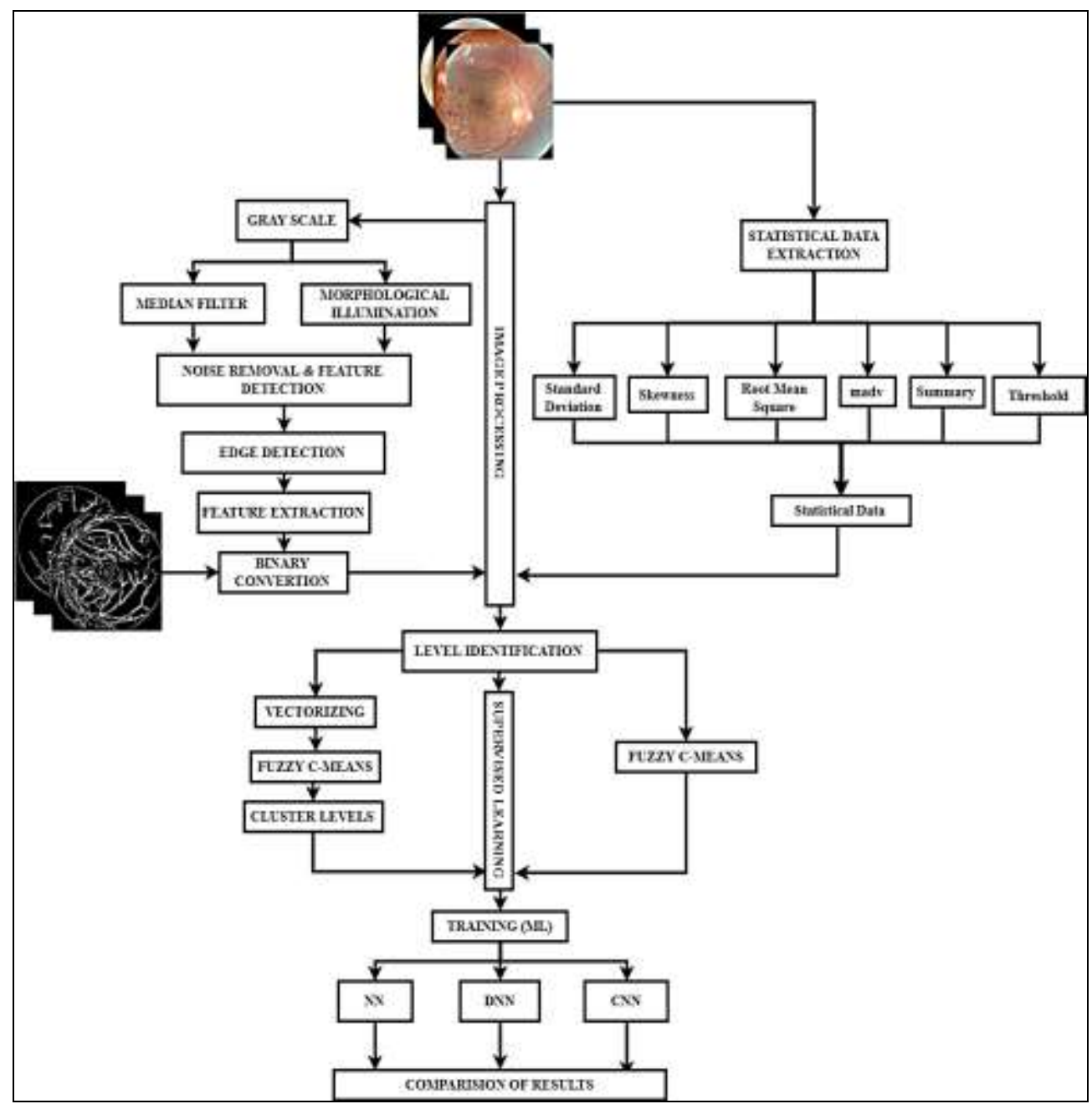

Figure 1. Model Architecture of Diabetic Retinopathy Analysis

Median Filter: In the day to day applications data acquisition can be done in many forms such as from sensors, images, satellite, logs and more. While in data acquisition there might be sparse \& noise existed. This form of data can't be utilized for any machine learning or research purpose. Camera images might contain lot of noise in the form of distortion, blur and many more. So, median filter is the one of the method to remove the noise and enhance the images for better extraction of features from the images for machine learning or artificial intelligence applications. In Figure 3first left image is noisy retina middle image shows filtered image and last right shows noise existed in the retina. The filter in consideration is a non-linear digital filtering. Each pixel in the image is replaced with the median value of the surrounding pixel values. This method we can preserve edges more precisely compared to the other filter methods. Here we can visualize white lesions.

Edge detection: It is the one of the feature extraction methods where the boundaries of the features are highlighted (i.e., the edges of the blood clots, white lesions, veins) are detected based on the sudden change in the pixel values with the neighborhood pixel intensity. Then the detected pixels will be assigned as the value ' 1 ' and the rest of all the pixels in the image or the whole matrix will be assigned as ' 0 '. By this method features can be identified in proper way. The edge detected images is a $2 \mathrm{D}$-matrix, which in turn can be converted to corresponding single array or $1 \mathrm{D}$-array vector. The matrix conversion takes place in such a way that all the row array values are placed side by side from start to end position without changing the sequence. The method adapted for detecting the edge 
detection is canny edge method. This method detects any abnormal changes in the pixels weights (intensity values) like crusts, troughs, color change etc.

$$
H_{\mathrm{ij}}=\frac{1}{2 \pi \sigma^{2}} \exp \left(\frac{(i-(k+1))^{2}+(j-(k+1))^{2}}{2 \sigma^{2}}\right) ; 1 \leq i, j \leq(2 k+1)
$$

Intensity gradient of the image can be calculated by the below mentioned formula,

$$
\begin{aligned}
G & =\sqrt{G_{x}^{2}+G_{y}^{2}} \\
\theta & =\operatorname{atan} 2\left(G_{y}, G_{x}\right)
\end{aligned}
$$

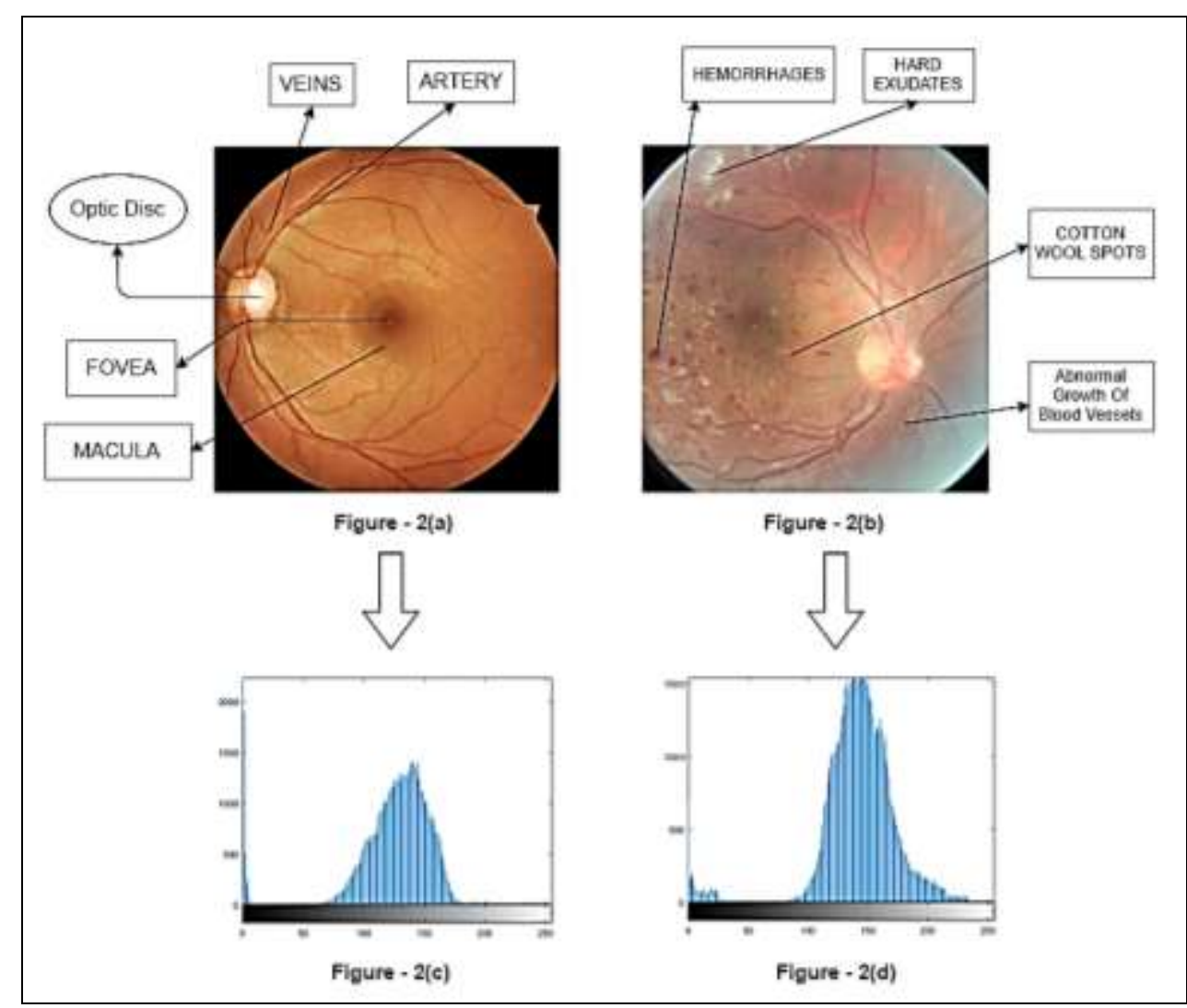

Figure 2. Comparison of Proliferative diabetic retinopathy (PDR) and Healthy Retinal Image

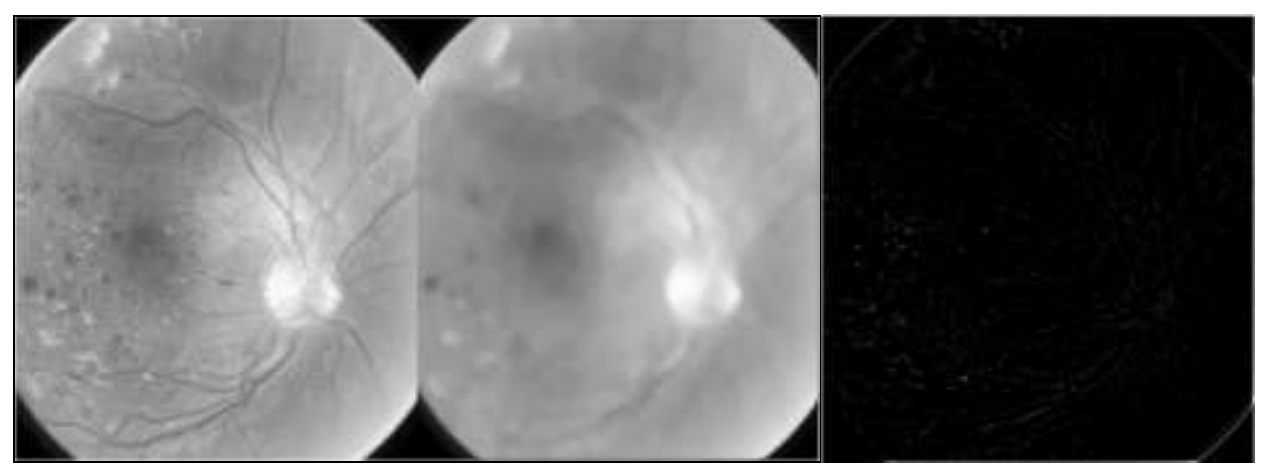

Figure 3. Non Filtered Image, Median Filter Image and Detected Noise out of the Image 


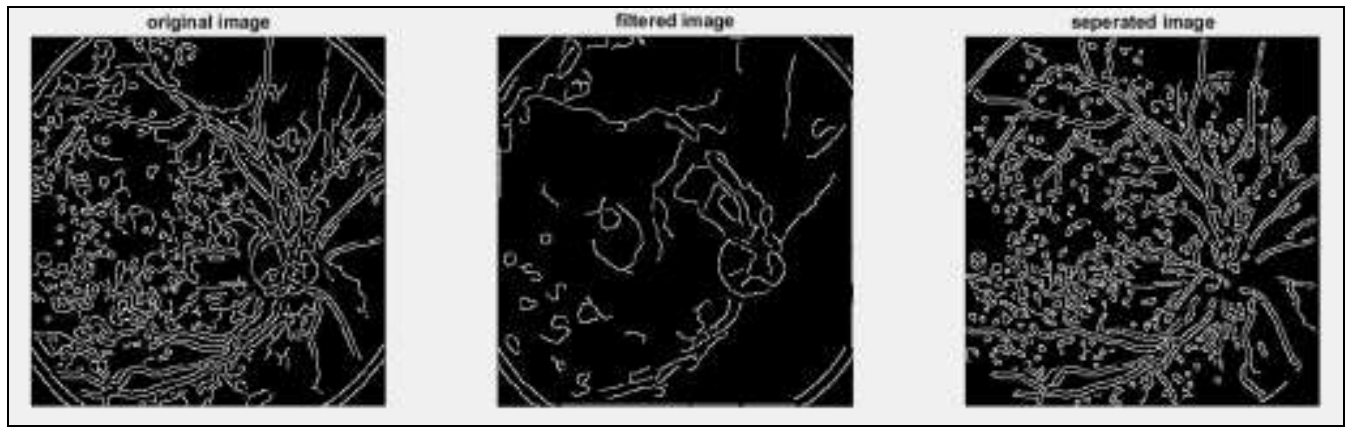

Figure 4. Levels of Edge Detection Method

Morphological non-uniform illusion: It is a feature enhancement technique, where a series of steps to be followed while enhancing the image. Where the brighter background is detected. This bright background is deleted from the original image to find the features in much better manner. The estimated background is shown in a 3D-graph (Figure 5)

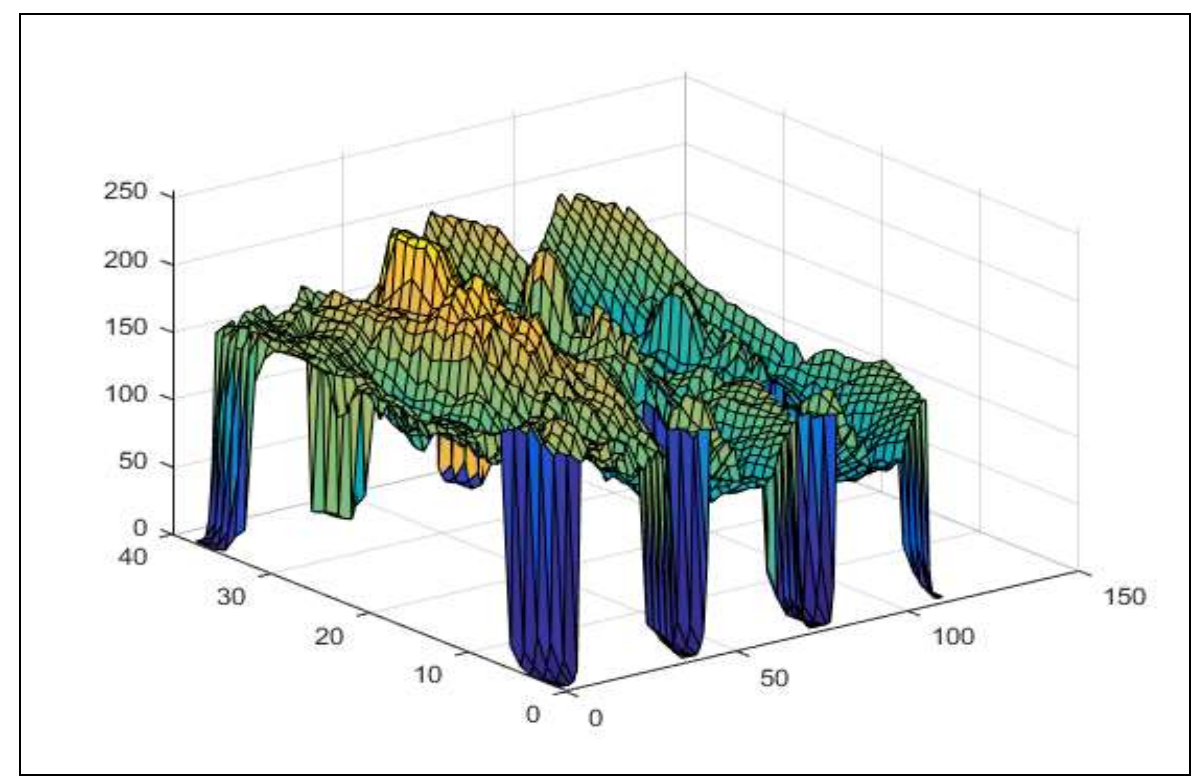

Figure 5. 3D Histogram Plotting of 3Band image

By subtracting the background illuminated image from the original image noised features are observed. Adjusting the image contrast and binarizing the image with the proper threshold noise from the image has been removed. This binary image is converted to RGB, where all the features are clearly visible. The step by step methodology results are displayed in the Figure 9.

\subsection{Feature Extraction}

Statistical approach: The various statistical terms used as the features for training the machine learning model is average, median, standard deviation, skewness, root mean square error, mean absolute deviation, quartiles, minimum, maximum \& threshold level values: 
Table 2. Features Extracted as Statistical Measure's

\begin{tabular}{|c|c|}
\hline Features & Relation of Each Feature With Respect to images \\
\hline Average & $\begin{array}{l}\text { The mean or average is the value which gives the information } \\
\text { about the central tendency of the given data i.e., 1D,2D etc. }\end{array}$ \\
\hline Median & $\begin{array}{l}\text { Over given range of values median separates the whole dataset } \\
\text { which can also termed as center of the whole data }\end{array}$ \\
\hline Standard deviation & $\begin{array}{l}\text { This provides details of how the values changes or differ from } \\
\text { average or mean value }\end{array}$ \\
\hline Skewness & $\begin{array}{l}\text { Skewness is used to calculate the lack of symmetry for the } \\
\text { probability distribution of a random variables. It is measured in- } \\
\text { terms of positive, negative \& normal }\end{array}$ \\
\hline $\begin{array}{l}\text { Root Mean Square } \\
\text { Error }\end{array}$ & $\begin{array}{l}\text { It used to find the difference between the value or the intensity } \\
\text { values of the pixel in the image }\end{array}$ \\
\hline $\begin{array}{l}\text { Mean Absolute } \\
\text { Deviation }\end{array}$ & $\begin{array}{l}\text { It is the average distance between each and every pixel values in } \\
\text { the data set from the mean or average }\end{array}$ \\
\hline Quartiles & $\begin{array}{l}\text { Over the range of pixel values is subdivided into } 4 \text { equal groups } \\
\text { according to the distribution of the pixel values }\end{array}$ \\
\hline Maximum & $\begin{array}{l}\text { It the maximum value or the largest value present in the whole } \\
\text { set of pixels }\end{array}$ \\
\hline Minimum & $\begin{array}{l}\text { It is the minimum value or the least value present in the whole } \\
\text { set of pixels }\end{array}$ \\
\hline Threshold & $\begin{array}{l}\text { The numerical value which segments the image \& responsible to } \\
\text { convert original or grey scale image into binary image }\end{array}$ \\
\hline
\end{tabular}

\subsection{Fuzzy C-Means}

The procesed images may have some feature loss during the resizing or filtering. The image levels can get changed because of that. Fuzzy C-means is a standard clustering mechanism works very well to identify the membership $\left(\mu_{\mathrm{ij}}\right)$ information form the image[19]. The cluster center updatation formula:

$$
\mu_{i j}=\frac{1}{\sum_{k=1}^{c}\left(\frac{d_{i j}}{d_{i k}}\right)^{(2 / m-1)}}
$$

And the centers $\left(\mathrm{v}_{\mathrm{j}}\right)$ gets updated according to:

$$
v_{j}=\frac{\sum_{i=1}^{n}\left(\mu_{i j}\right)^{m} x_{i}}{\sum_{i=1}^{n}\left(\mu_{i j}\right)^{m}}, \forall j=1,2, \ldots c
$$

By using FCM clusterng the given level and clustered image levels have been compared to make the training levels valid. The idea behind using FCM is because of the data points are not mandatory to be under one cluster center, here data points are given to 
the membership of each cluster centers, because of this nature one data point can be under multiple cluster centers[20][21].

\subsection{Neural Networks with Multilayer Perceptron}

The first NN model considered is NN with multilayer perceptron. It's a basic NN model having multiple layer of computational units inter connected via feed forward way [23]. The activation function used here is the "sigmoid" function[24]. The algorithm used is the traditional "Back Propagation" $f^{\prime}(x)=f(x)(1-f(x))$. The nurons for Perceptron modeling is being calculated through $\sqrt{\text { pixels }}$. The epoches has being selected based on multiple runs of the $\mathrm{NN}$ model, and as the model trained 268 epoches are considered as the approximately accurate. Figure 6 shown the perceptron model used for statistical data training.

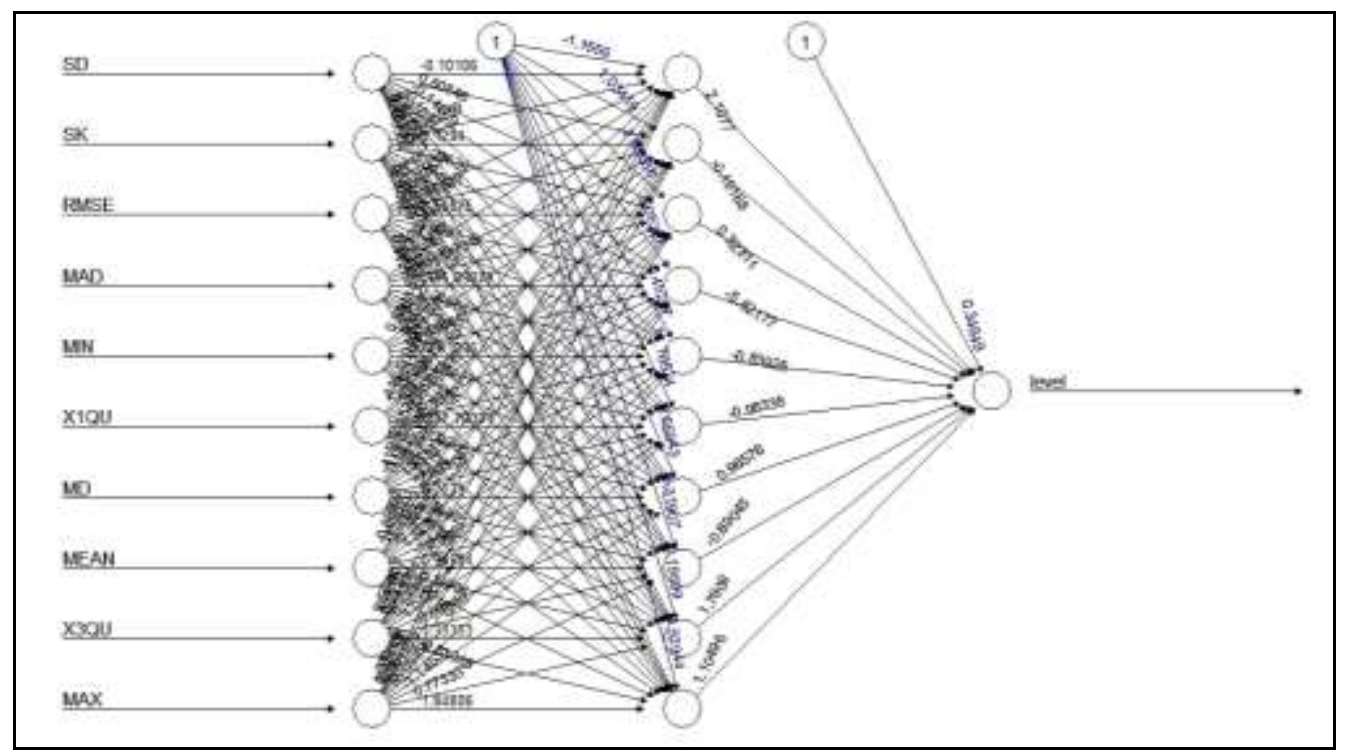

Figure 6. Neural Network Perceptron Model for Statistical Data

\subsection{Deep Neural Network (DNN)}

After validating the accuracy result of NN model with testing samples, the next DNN models have been implemented. The idea of apply this model is to check the accuracy with the NN model. Model accuracy definitely will differ because of utilizing with multiple hidden layer concerning input plus output[26][27]. The multiple layer extracts feature from lower level of layers and increases the potential of a small network[26]. In DNN model as algorithm "Back Propagation" is being used [31][32]. Some of the study suggested that even for Sparse network "Back Propagation" performs well [28]. The weight updated in back propagation via "stochastic gradient descent" algorithm,

$$
w_{i j}(t+1)=w_{i j}(t)+\eta \frac{\partial C}{\partial w_{i j}}+\varepsilon(t)
$$

Here, $\eta$ is assigned as learning rate, $\mathrm{C}$ is considered as cost function, and $\xi(\mathrm{t})$ a type of stochastic function. The method of DNN having three Fully Connected (FC) layer. Two activation layer with "ReLU" as activation function, for building model "Tanh" and "Sigmoid" activation functions also but "ReLU" performs better thn other two approaches. "ReLU" uses smooth rough calculation to the rectifier, $f(x)=\ln \left(1+e^{z}\right)$. The main activation function defined as $f(x)=\max (0, x)$. The ReLU considered as the most popular activation function as for Computer Vision [28][29]. The DNN model uses one softmax layer or normalized exponential function as, 
$p_{j}=\frac{\exp \left(x_{j}\right)}{\Sigma_{k} \exp \left(x_{k}\right)}$ where, Pj represents the class probability (output of the unit $\mathrm{j}$ ) and $\mathrm{xj}$ and $\mathrm{xk}$ represent the total input to units $\mathrm{j}$ and $\mathrm{k}$ of the same level respectively. Cost function defined as, $C=-\sum_{j} d_{j} \log \left(P_{j}\right)$ here, dj represents the target probability for output unit $\mathrm{j}$ and $\mathrm{Pj}$ is the probability output for $\mathrm{j}$ after applying the activation function[30].Both $\mathrm{NN}$ and DNN has been carried out on both statistical data of diabetic images and also processed retinopathy images. The difference is coming for the training time of the of both techniques. During work out sessions with DNN, statistical data taken less time then image data. Figure 7 showing the deep learning model used in training of image data.

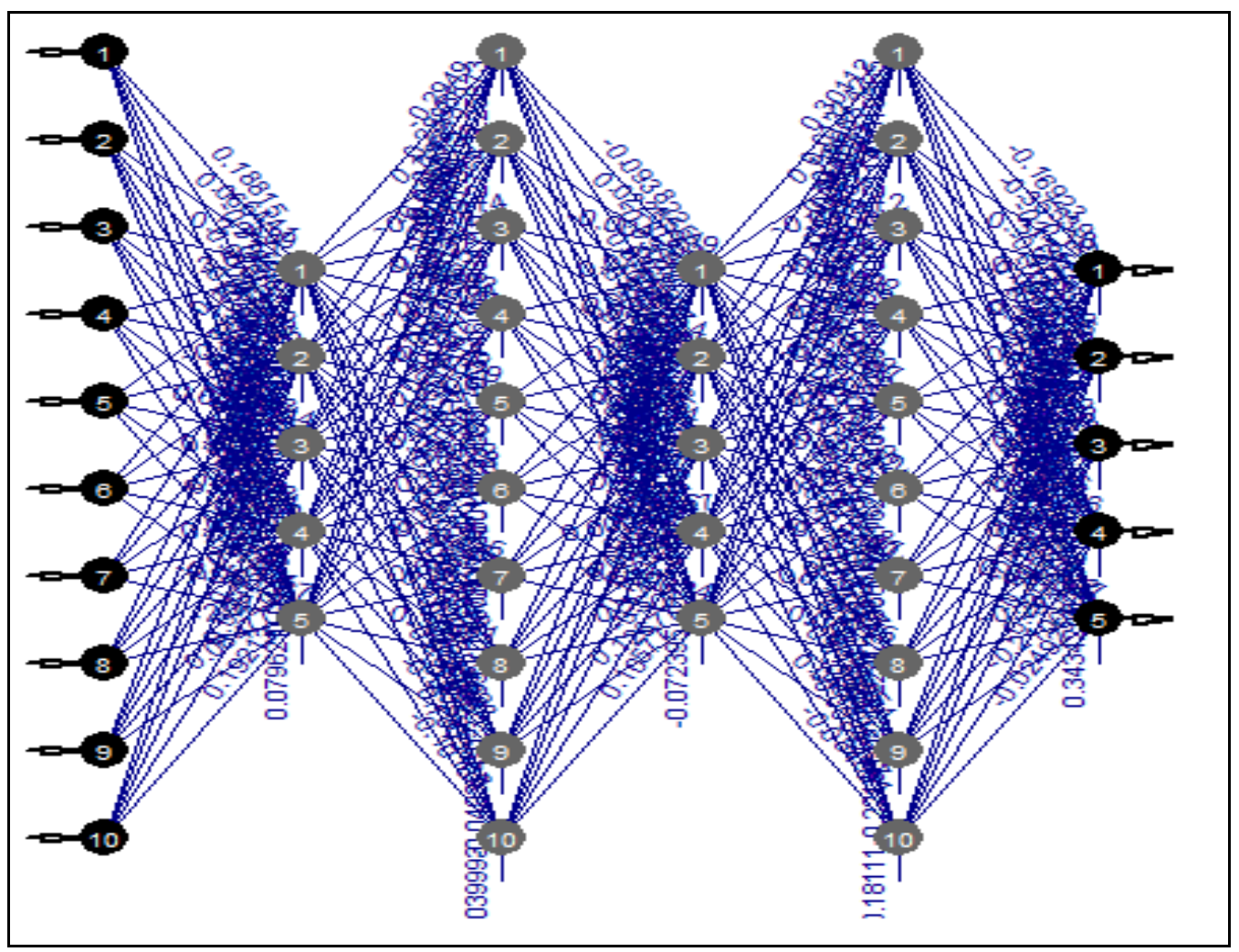

Figure 7. Structure of Deep Neural Network

\subsection{Convolutional Neural $\operatorname{Network}(\mathrm{CNN})$}

Only image data is being trained for CNN model. Processed images with single band has been given as a input of the network with given levels. The CNN model has been considered is the VGGnet model. The VGGnet [39] model structured of CONV layers which performs $3 \times 3$ convolution alongside stride: 1 and pad:1, and of POOL layers which performs $2 \times 2$ maxpooling with stride: 2 . There is no padding existed in the network. The network trained with CPU support. As activation function ReLU has been used. All convolutional layers are followed with Maxpool used in the pooling layed for extracting the most significant feature between the image pixel. VGGnet works very well with densed featured images. As per model there is no normalizaton layers used here, because either way it does not improve the accuracy of the model. Figure 8 giving the model view. 


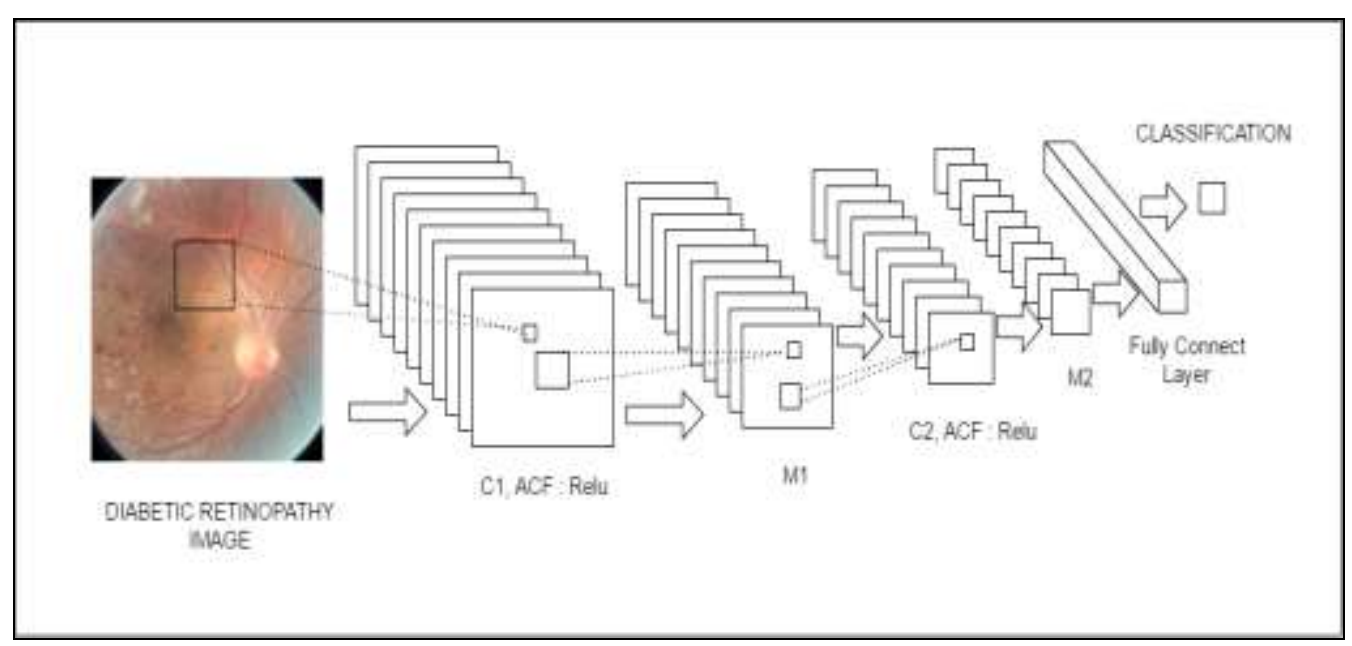

Figure 8. CNN Model for Image Classification

\section{Results and Discussions}

The result/outcome of any image preprocessing method or technique is enhanced image with rich features in the from the Figure 9 in order to get the enhanced image a step by step procedure need to be followed where result of a step is feed as input to the next step for the feature extraction. From the actual/original image by removing high intensity background noise in the image can be reduced in the mean while features with large difference in the pixel intensity values are highlighted. This processed image is converted to RGB image which gives the better visual information of a particular image. RGB image then separated into all the 3 types of layers as RED, GREEN \& BLUE. Where GREEN layer is the output of this image preprocessing technique highlighting the information about the exudates, white lesions, veins. Figure 9 shows the steps of morphological image processing carried out in the study.

Both the statistical image data and processed images are trained with BNN, DNN models. For both models 1000 retinopathy images have been considered. Because for single layer hidden layer falls effect of poor training in BNN model. DNN model takes less time also accuracy of training is good compared to BNN model.

For both statistical data and processed image data DNN gives good accuracy for the hyperparmeter adjustment. CNN considered as highly rated model for image vision, model trained with 1000 images having accuracy of $72.5 \%$ with VGGnet model. The model trained with CPU support which made an impact on training time. For testing the model, 300 images have been considered, as for result concerns both the normal retinopathy images and Proliferative diabetic retinopathy images have predicted perfectly by the model. Mostly misclassification happened for level 1-2 level images. Figure 10 shows the probabilistic result of classification of images with CNN model. In Table 3 detailed parameter utilization have been listed. 


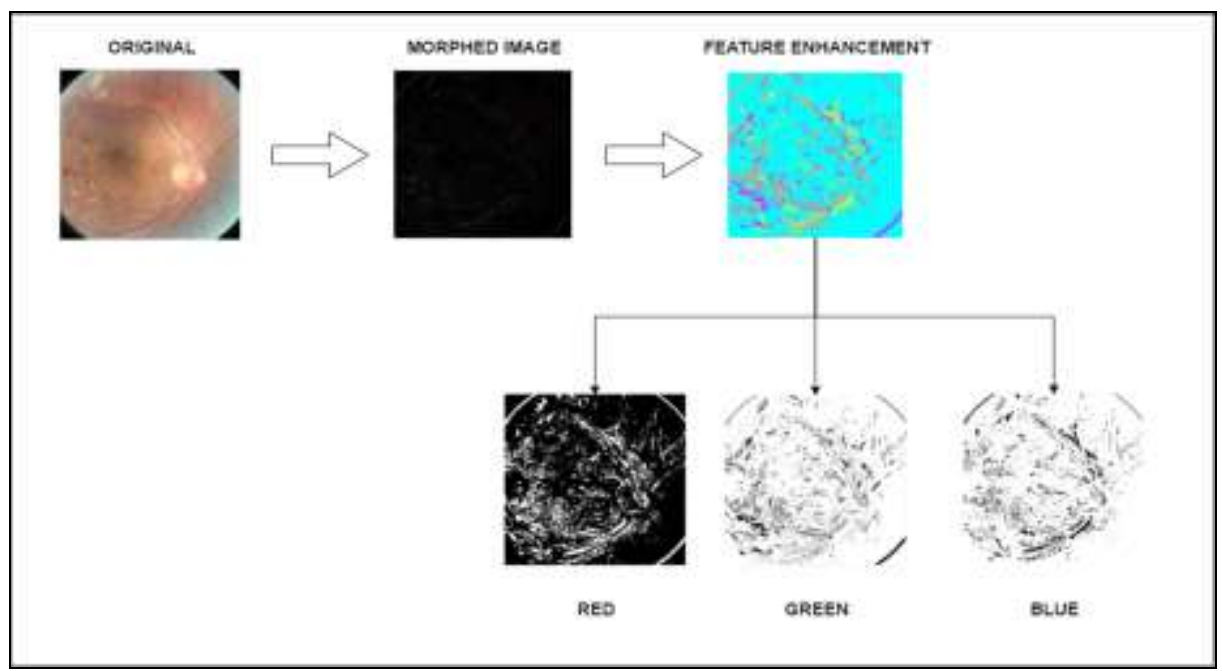

Figure 9. Morphological Segmenting Stages

Table 3. Parameter used for Training of Neural Network Models

\begin{tabular}{|l|l|l|l|}
\hline & BNN & DNN & CNN (VGGnet) \\
\hline Activation Function & Sigmoid & ReLU & ReLU \\
\hline Fully Connected Layer & - & 3 & 3 \\
\hline Convolution Layer & - & - & 16 \\
\hline Learning Rate & 0.2 & 0.2 & 0.5 \\
\hline Epoches & 200 & 200 & 200 \\
\hline Time (On CPU Approx) & $1 \mathrm{hr}$ & $15 \mathrm{~min}$ & $1.5 \mathrm{hr}$ \\
\hline Image Resolution & $300 \times 300$ & $300 \times 300$ & $300 \times 300$ \\
\hline
\end{tabular}

When a new image is feed into the CNN model for the prediction it gives the results in the probabilistic results, such as probability that an image can be classified into various classes. The above figure shows the results of the different images each at different stage $\&$ their probabilities. As observed for $0,1,4$ - levels the probability prediction of desired class gives more than 50\%. The rest of the score is distributed among the rest of the classes. But where as in $2 \& 3$ levels the probability values of all the classes are less than $50 \&$ nearly equal which is the main draw back while classification of these two classes \& main cause for the less accuracy of the model. For testing validation in all models RMSE (Root Mean Square Error) have been calculated as for error concerns BNN getting high error rate for detection whereas DNN performing better for both statistical data model as well as image data models. But for CNN poor training leads to high error rate compared to DNN model. Table 2 shows the training and testing accuracy of all the models. 


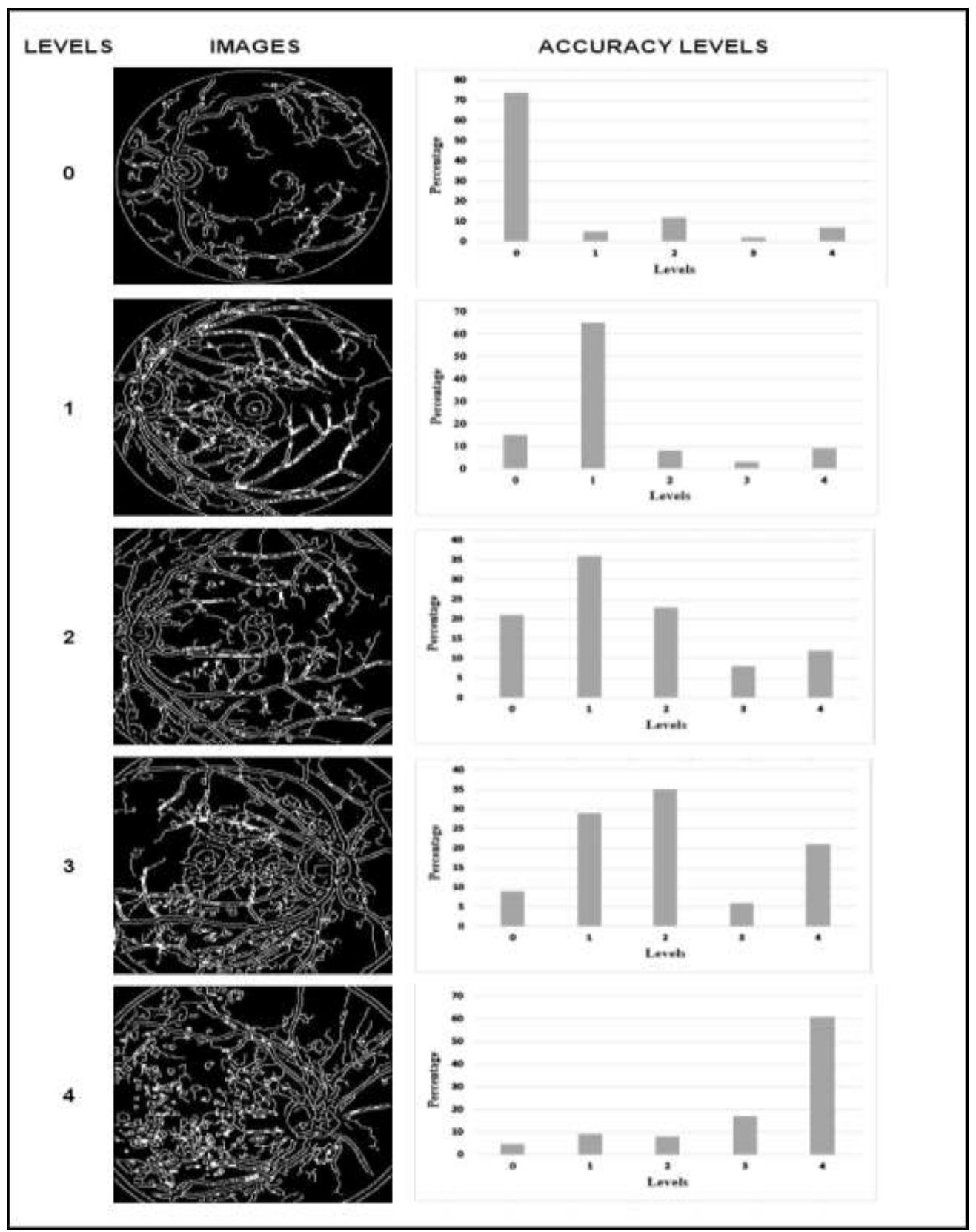

Figure 10. Probabilistic Result of Image Class Identification

Table 4. Accuracy Percentages of Each Algorithm for Processed Image Classification Image

\begin{tabular}{|l|l|l|}
\hline & TRAINING & TESTING \\
\hline BNN & 62.7 & 42 \\
\hline DNN & 89.6 & 86.3 \\
\hline CNN (VGGNET) & 76.4 & 78.3 \\
\hline
\end{tabular}


Table 5. Accuracy Percentages of Each Algorithm for Statistical Data of the Image

\begin{tabular}{|l|l|l|}
\hline & TRAINING & TESTING \\
\hline BNN & 45.7 & 35.6 \\
\hline DNN & 84.7 & 82.3 \\
\hline
\end{tabular}

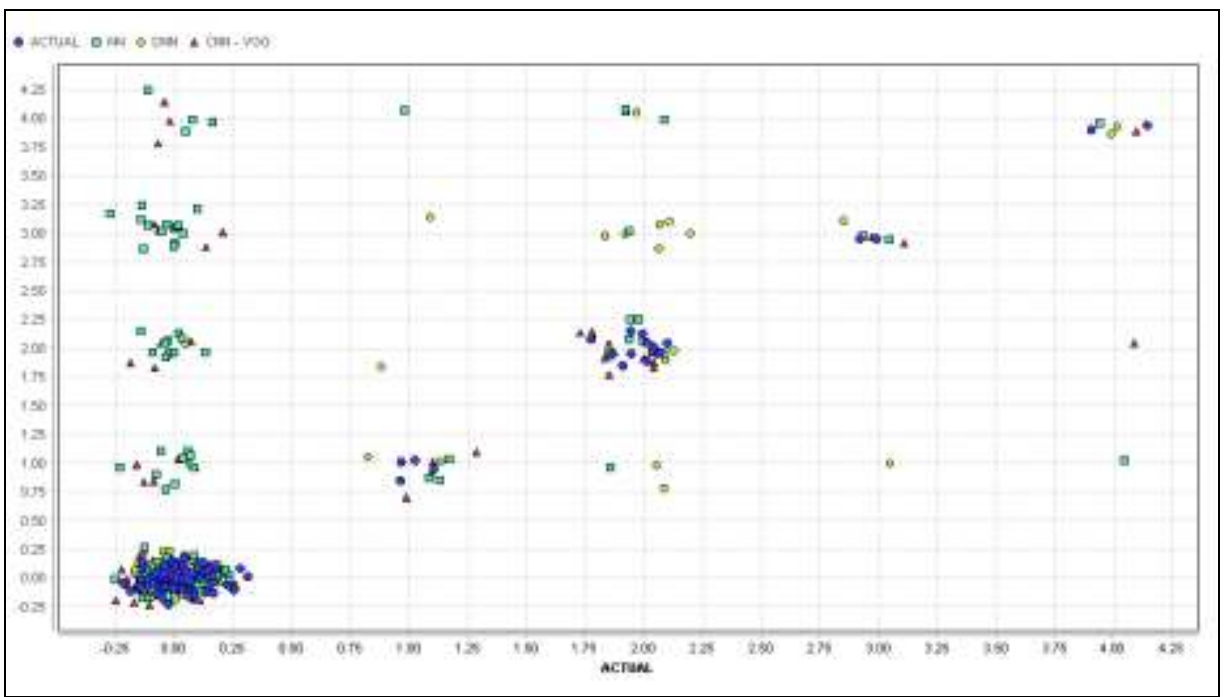

Figure 11. Plotting of Actual vs Predicted Results $(x-$ axis Shows the Actual Levels of the Image, $y$-axis Shows the Predicted Levels of the image as for FNN DNN and CNN Models Concern)

The above figure shows the classification of the various models results with the actual results. Legends provides the information of various scatters plotted. Cluster density of the actual results for 0th Level is high, followed with 2nd, 1st, 3rd \& 4th levels. Miss Classification of 0th level for the NN model (green color) is more, where mostly it is classified as 2 nd \& 3 rd levels. For the rest of the levels deviation of miss classification is less compared to the 0th level. Miss classification of DNN at 2nd level is more, for rest of the classes it is giving good classification resulting the highest accuracy in our model. Where as in CNN Oth level misclassification is more rather than other levels but not worse than the neural network model.

Figure 12, gives the comparison results of all the images (100 numbers) predicted by the different models. Each color displayed represents the different model $\&$ its related results plotted by using the line graph. Results at a point provides the information about all the predictions of NN, DNN \& CNN models including the actual value. Hence in this way result comparison can be done for each and every individual image used for training the model \& calculates the validation accuracy. As from our study from Figure 11 and Figure 12 it's visible that DNN result is more or less close to the actual image levels. 


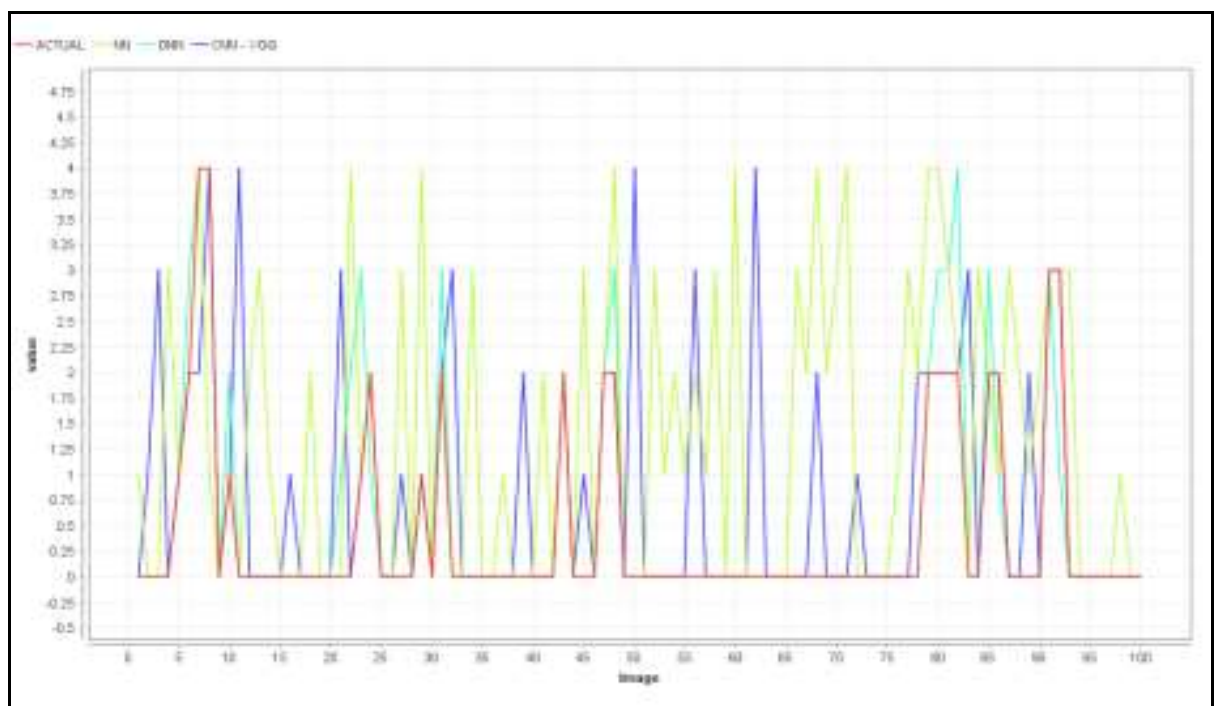

Figure12. Line Plot of Actual and Predicted Results (X-Axis Shows Image Numbers and Y-Axis Shows the Predicted Image Levels as Well As Actual Image Levels)

\section{Conclusions}

The paper is about proposing an optimal model for Diabetic Retinopathy detection. Processing of Retinopathy images is very essential to get proper features. Statistical values can predict level of severity properly but in case of noisy images the chances of getting poor data will lead to lower accuracy. For getting better result selecting for proper features out of the image also important. Both CNN and DNN models are effective in term for image, because of CPU training time of CNN getting affected in the study, in this case DNN outperforms CNN for training accuracy as well as validation accuracy. For future work model can train with GPU system, with more number of processed data for getting higher accuracy result. A standalone application will be good for identification of retinopathy images. Also, the proposed model can be integrated with existing NPDR screening algorithms in [40] for enhanced prioritization and resourcefulness of the present day eye-care delivery.

\section{References}

[1] V. Raman, P. Then and P. Sumari. "Proposed retinal abnormality detection and classification approach: Computer-aided detection for diabetic retinopathy by machine learning approaches", Communication Software and Networks (ICCSN), 2016 8th IEEE International Conference on. IEEE, (2016).

[2] N. Singh and R. Chandra Tripathi. "Automated early detection of diabetic retinopathy using image analysis techniques", International Journal of Computer Applications, vol. 8, no. 2, (2010), pp. 18-23.

[3] T. A. Soomro, "Role of Image Contrast Enhancement Technique for Ophthalmologist as Diagnostic Tool for Diabetic Retinopathy", Digital Image Computing: Techniques and Applications (DICTA), 2016 International Conference on. IEEE, (2016).

[4] Y. Zhao, "Intensity and Compactness Enabled Saliency Estimation for Leakage Detection in Diabetic and Malarial Retinopathy", IEEE Transactions on Medical Imaging, vol. 36, no. 1, (2017), pp. 51-63.

[5] D. K. Prasad, L. Vibha and K. R. Venugopal, "Early detection of diabetic retinopathy from digital retinal fundus images", Intelligent Computational Systems (RAICS), 2015 IEEE Recent Advances in. IEEE, (2015).

[6] M. U. Akram, "Detection and classification of retinal lesions for grading of diabetic retinopathy", Computers in biology and medicine, vol. 45, (2014), pp. 161-171.

[7] R. J. Winder, "Algorithms for digital image processing in diabetic retinopathy", Computerized Medical Imaging and Graphics, vol. 33, no.8, (2009), pp. 608-622.

[8] Mu. S. Haleem, "Automatic extraction of retinal features from colour retinal images for glaucoma diagnosis: A review", Computerized medical imaging and graphics, vol. 37, no. 7, (2013), pp. 581-596. 
[9] G. G. Gardner, "Automatic detection of diabetic retinopathy using an artificial neural network: a screening tool", British journal of Ophthalmology, vol. 80, no. 11, (1996), pp. 940-944.

[10] R. Sivakumar, "Diabetic retinopathy analysis", BioMed Research International, vol. 2005, no. 1, (2005), pp.20-27.

[11] S. Roychowdhury, D. D. Koozekanani and K. K. Parhi, "Dream: Diabetic retinopathy analysis using machine learning", IEEE journal of biomedical and health informatics, vol. 18, no. 5, (2014), pp. 17171728 .

[12] M. N. Wernick, "Machine learning in medical imaging", IEEE signal processing magazine, vol. 27, no. 4, (2010), pp. 25-38.

[13] T. R. Rakshitha, D. Devaraj and S.C. Prasanna Kumar, "Comparative study of imaging transforms on diabetic retinopathy images", Recent Trends in Electronics, Information \& Communication Technology (RTEICT), IEEE International Conference on. IEEE, (2016).

[14] H. H. Vo and A. Verma, "Discriminant color texture descriptors for diabetic retinopathy recognition", Intelligent Computer Communication and Processing (ICCP), 2016 IEEE 12th International Conference on. IEEE, (2016).

[15] P. Patil, "An efficient method of detecting exudates in diabetic retinopathy: Using texture edge features", Advances in Computing, Communications and Informatics (ICACCI), 2016 International Conference on. IEEE, (2016).

[16] R. Williams, "Epidemiology of diabetic retinopathy and macular oedema: a systematic review", Eye, vol. 18, no. 10, (2004), pp. 963-983.

[17] J. C. Bezdek, R. Ehrlich and W. Full, "FCM: The fuzzy c-means clustering algorithm”, Computers \& Geosciences, vol. 10, no. 2-3, (1984), pp. 191-203.

[18] P.J. Saine and M. E. Tyler, "Ophthalmic photography: a textbook of fundus photography", angiography, and electronic imaging. Butterworth-Heinemann, (1997).

[19] K.-S. Chuang, "Fuzzy c-means clustering with spatial information for image segmentation", computerized medical imaging and graphics, vol. 30, no. 1, (2006), pp. 9-15.

[20] Fuzzy c-means by Balaji K and Juby N Zacharias.

[21] W. Cai, S. Chen and D. Zhang, "Fast and robust fuzzy c-means clustering algorithms incorporating local information for image segmentation", Pattern recognition, vol. 40, no. 3, (2007), pp. 825-838.

[22] C. Sinthanayothin, "Automated localisation of the optic disc, fovea, and retinal blood vessels from digital colour fundus images", British Journal of Ophthalmology, vol. 83, no. 8, (1999), pp. 902-910.

[23] K. Hornik, M. Stinchcombe and H. White, "Multilayer feedforward networks are universal approximators", Neural networks, vol. 2, no. 5, (1989), pp. 359-366.

[24] G. Cybenko, "Approximation by superpositions of a sigmoidal function", Mathematics of Control, Signals, and Systems (MCSS), vol. 2, no. 4, (1989), pp. 303-314.

[25] R. Hecht-Nielsen, "Theory of the backpropagation neural network", Neural Networks, vol. 1. Supplement-1, (1988), pp. 445-448.

[26] Y. Bengio, "Learning deep architectures for AI", Foundations and trends® in Machine Learning, vol. 2, no. 1, (2009), pp.1-127.

[27] J. Schmidhuber, "Deep learning in neural networks: An overview”, Neural networks, vol. 61, (2015), pp. 85-117.

[28] Y. LeCun, Y. Bengio and G. Hinton, "Deep learning”, Nature 521.7553 (2015), pp. 436-444.

[29] Glorot, Xavier, Antoine Bordes, and YoshuaBengio", Deep Sparse Rectifier Neural Networks. Aistats. vol. 15 , no. 106, (2011).

[30] G. Hinton, "Deep neural networks for acoustic modeling in speech recognition: The shared views of four research groups", IEEE Signal Processing Magazine, vol. 29, no. 6, (2012), pp. 82-97.

[31] H. J. Kelley, "Gradient theory of optimal flight paths", Ars Journal, vol. 30, no. 10, (1960), pp. 947-954.

[32] A. E. Bryson, "A gradient method for optimizing multi-stage allocation processes", Proc. Harvard Univ. Symposium on digital computers and their applications, (1961).

[33] R. Casanova, S. Saldana, E. Y. Chew, R. P. Danis, C. M. Greven and W. T. Ambrosius, "Application of Random Forests Methods to Diabetic Retinopathy Classification Analyses", PLOS ONE, vol. 9, no. 6, (2014), pp. 1-7.

[34] C. Sinthanayothin, J.F. Boyce, T.H. Williamson, H.L. Cook, E. Mensah, S. Lal and D. Usher. "Automated Detection of Diabetic Retinopathy on Digital Fundus Images", Diabetic Medicine, vol. 19, (2002), pp. 105-12.

[35] D. Usher, M. Dumskyjs, M. Himaga, T.H. Williamson, S. Nussey and J. Boyce, "Automated Detection of Diabetic Retinopathy in Digital Retinal Images: A Tool for Diabetic Retinopathy Screening", Diabetic Medicine, vol. 21, (2003), pp. 84-90.

[36] H. F. Jaafar, A. K. Nandi and W. Al-Nuaimy, "Automated Detection And Grading Of Hard Exudates From Retinal Fundus Images", 19th European Signal Processing Conference (EUSIPCO), (2011), pp. 66-70.

[37] "National Diabetes Statistics Report, 2014." Centers for Disease Control and Prevention. January 1, 2014. Accessed December 26, (2014).

[38] "Diabetes", World Health Organization. November 1, 2014. Accessed December 26, (2014). http://www.who.int/mediacentre/factsheets/fs312/en/. 
[39] K. Simonyan and A. Zisserman, "Very deep convolutional networks for large-scale image recognition", arXiv preprint arXiv, vol.1409, no. 1556, (2014).

[40] S. Roychowdhury, D. Koozekanani and K. Parhi, "Dream: Diabetic retinopathy analysis using machine learning", IEEE Journal of Biomedical and Health Informatics, vol. 18, no. 5, (2014), pp. 1717-1728.

[41] M. R. K. Mookiah, "Computer-aided diagnosis of diabetic retinopathy: A review", Computers in biology and medicine, vol. 43, no. 12, (2013), pp. 2136-2155.

[42] NCD Risk Factor Collaboration, "Worldwide trends in diabetes since 1980: a pooled analysis of 751 population-based studies with 4. 4 million participants", The Lancet, vol. 387, no. 10027, (2016), pp. 1513-1530.

[43] C. J. Murray and A. D. Lopez, "Mortality by cause for eight regions of the world: Global Burden of Disease Study", The lancet, vol. 349, no. 9061, (1997), pp. 1269-1276.

[44] Early Treatment Diabetic Retinopathy Study Research Group, "Fundus photographic risk factors for progression of diabetic retinopathy: ETDRS report number 12", Ophthalmology, vol. 98, no. 5, (1991), pp. 823-833.

\section{Authors}

Suvajit Dutta, Bonthala CS Manideep, they are both M. Tech. graduate studens with specialization in Big Data Analytics from Vellore Institute of Technology. Their interest is in Deep learning and Data Science. Currently they are working in the field of Image Processing.

Syed Muzamil Basha, he had his Bachelor of Science in Information Technology at SITAMS, MTech in Information Technology (Networking) at VIT University and currently doing his research at VIT University. His research area are Wireless Sensor Networks, Text Mining and Big Data Predictive Analytics.

N. Ch. S. N. Iyengar (b 1961), he currently Professor at the Sreenidhi Institute of Science and Technology (SNIST) Yamnapet, Ghatkesr, Hyderabad, Telengana, India. His research interests include Agent-Based Distributed Computing, Intelligent Computing, Network Security, Secured Cloud Computing and Fluid Mechanics. He had 32+ years of experience in teaching and research, guided many scholars, has authored several textbooks and had nearly 200+ research publications in reputed peer reviewed international journals. He served as PCM/reviewer/keynote speaker/ Invited speaker. He received 2017 achievement award for his contributions in teaching and research by IOSRD.

Ronnie D. Caytiles, he had his Bachelor of Science in Computer EngineeringWestern Institute of Technology, Iloilo City, Philippines, and Master of Science in Computer Science- Central Philippine University, Iloilo City, Philippines. He finished his Ph.D. in Multimedia Engineering, Hannam University, Daejeon, Korea. Currently, he serves as an Assistant Professor at Multimedia Engineering department, Hannam University, Daejeon, Korea. His research interests include Mobile Computing, Multimedia Communication, Information Technology Security, Ubiquitous Computing, Control and Automation 